\section{Temporal factors influencing performance in the missing scan paradigm*}

\author{
JOHN JONIDES†, STANLEY C. COLLYER††, and WILLIAM BEVAN \\ The Johns Hopkins University, Baltimore, Maryland 21218
}

Sixty Ss were each presented 120 random sequences of 9 of the 10 digits (0-9); their task on each trial was to supply the missing digit. Two stimulus input rates and two response time limits were combined factorially to yield four experimental conditions. Contrary to expectation, evidence seeming to support the use of a strategy based on the ordinal properties of the stimuli was found only when rapid $(1-\mathrm{sec})$ responses were required, regardless of input rate. It is suggested that this result is most reasonably attributed to the operation of a guessing bias for numbers in the middle of the ordinal sequence. Differences in the constitution of the stimulus series may account for the failure to confirm previous findings of an ordinal strategy.

The temporal course of the processes of encoding, storage, and retrieval has recently been of considerable interest in studies of short-term memory (Aaronson, 1967). One type of methodological tool that has been developed for use in such studies concerns the partial report of items in memory (e.g., Sperling, 1960; Averbach \& Coriell, 1961). Procedures involving partial report are particularly well suited for studies of memory capacity, since they place relatively light demands upon response processes.

The missing scan procedure (Buschke, 1963a) is one such partial report technique. In this paradigm, $S$ is presented, in random order, $n-1$ items (usually numbers) from a known stimulus set of size $n$; his task is simply to report the missing item. Since a correct response presumably depends upon $S$ 's retention of information concerning all items, an assessment can be made of his storage capabilities without being contaminated by the retrieval of multiple items. The missing scan technique has been used to study such topics as storage capacity (Buschke, 1963a), serial retention of items (Buschke, 1963b), and the effect of extraneous items on serial retention (Buschke \& Hinrichs, 1968).

While this task has been used as a methodological device for the study of basic memory phenomena, there are some interesting questions raised by the paradigm itself-for example, what is the nature of the encoding and

* This research was supported by Contract N00014-67-A-0163-0001 between The Johns Hopkins University and the Physiological Psychology Branch, Office of Naval Research.

+ Now at the University of Pennsylvania, Department of Psychology, 3813-15 Walnut Street, Philadelphia, Pennsylvania 19104.

+ Now at Technology Incorporated, Life Sciences Division, 8531 North New Braunfels Avenue, San Antonio, Texas 78217 . comparison operations $S$ typically performs upon the input in order to identify the absent item? Madsen and Drucker (1966) report that $S s$ are more likely to devise information reduction techniques to facilitate performance in a missing span task than in the ordinary digit scan paradigm. The nature of these techniques has been explored (Buschke \& Hinrichs, 1968; Buschke \& Lenon, 1969), and there is some evidence pointing to the use of an "ordinal strategy" - that is, one based upon the ordinal properties of the stimulus numbers. When errors in the missing scan task are analyzed according to their closeness to the correct answer on an ordinal scale, a gradient is found such that most errors occur relatively close to the correct answer. For example, if the missing number in a particular series is $14, \mathrm{~S}$ will be more likely to respond with the numbers 13 or 15 than with 25 . It has 1969) that the steepness of this gradient appears to depend upon the amount of time available to $S$ during presentation of the items: only when input speed was sufficiently slow did the S's strategy apparently involve making use of the ordinal properties of the numbers.

The present experiment further investigates the nature of the strategies people employ in the missing scan task. Both the length and the composition of the numerical series were modified from those previously also been found (Buschke \& Lenon, employed. Buschke commonly used stimulus sets of 13 elements, encompassing either the numbers 1-13 or 13-25. The present experiment employed the 10-digit set $0-9$, which had been found in preliminary work to permit rapid responses of considerably greater accuracy than those obtained with a 13-element set. The speed and accuracy made possible by this stimulus set seemed to indicate that, in some situations, the response is far too "automatic" to be mediated by a series of operations as complex as that implied by an ordinal strategy. In order to obtain information concerning those conditions under which an ordinal strategy would and would not obtain, input and response speeds were manipulated orthogonally. Since an effective ordinal strategy would seem to demand an ongoing reorganization of the items as they are presented, it was predicted that slow input would permit such a strategy but fast input would preclude it (Buschke \& Lim, 1967). It was further hypothesized that reasonable response speed limitations should not substantially hinder the operation of an ordinal strategy, but should have a major impact on those conditions not permitting the material to be reorganized during presentation.

\section{METHOD}

Two stimulus input rates of $1 / \mathrm{sec}$ and $4 /$ sec were combined with two response time limits of 5 and 1 sec, yielding a 2 by 2 design. Sixty male undergraduates of The Johns Hopkins University served as Ss and were paid for their participation. Fifteen Ss were assigned to each of the four conditions.

\section{Stimuli}

Each trial involved a random presentation of 9 of the 10 digits from the set $0-9$. A total of 120 sequences was constructed, with the constraints that each number serve as the correct (missing) digit equally often and that each digit appeax in each serial position an approximately equal number of times. The stimuli were presented auditorily by means of a tape recorder.

Five practice trials were given at the beginning of the session, and a short break was provided after each block of 


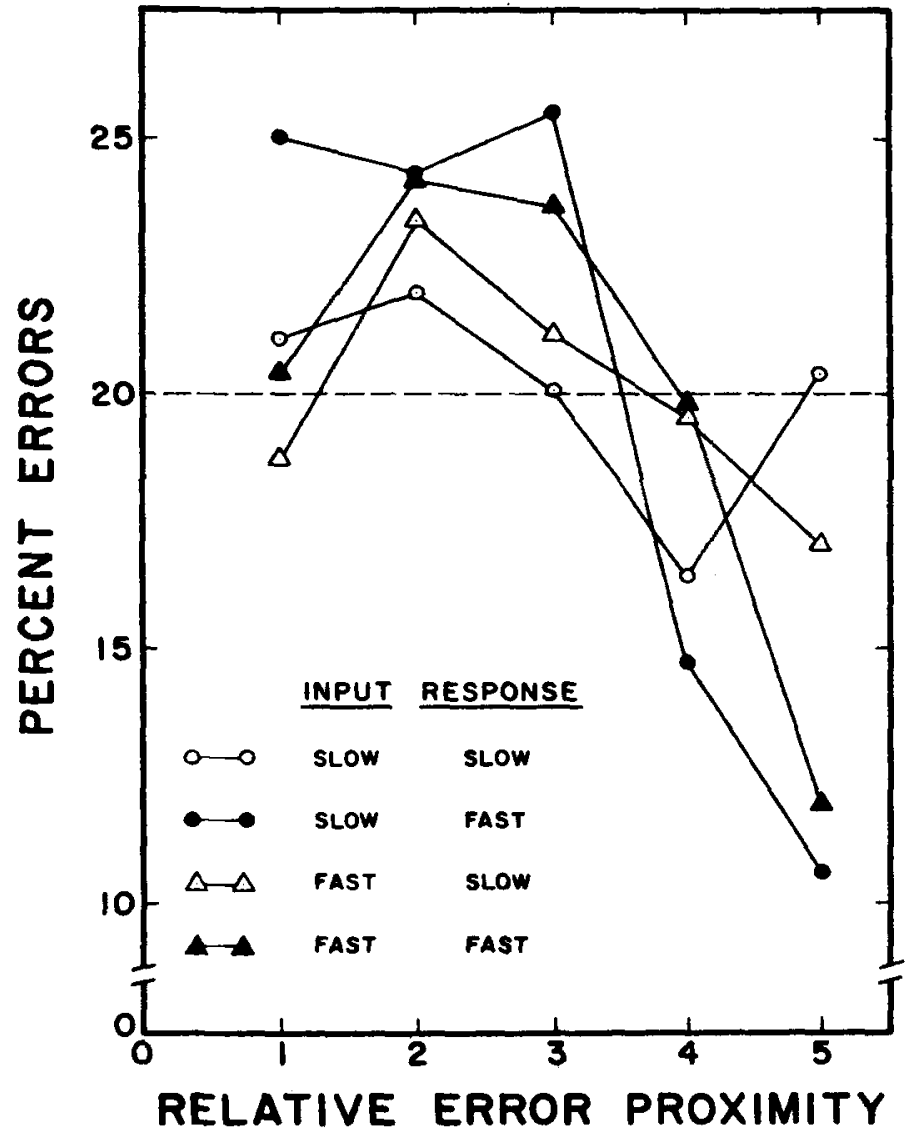

Fig. 1. Error proximity functions for the four experimental conditions.

20 trials. The session lasted about $50 \mathrm{~min}$.

\section{Procedure}

For each trial, a ready signal was given, followed by a 2-sec delay. The nine digits were then presented, and $S$ was instructed to indicate verbally the missing digit following the ninth number of the series. 1 There was a 10-sec interval between trials.

To insure that $S$ complied with the response time limit while performing as accurately as possible, a payoff schedule was constructed so that each correct response within the limit was rewarded with a $3 c$ bonus. However, $S$ lost $1 \mathrm{c}$ for both an incorrect response within the time limit and for a correct response that was too slow. Finally, 3c was lost for a slow, incorrect response. Information concerning accuracy and speed was provided after each trial.

During each trial, a metronome could be heard ticking in the background at 4 beats/sec. This provided both $S$ and $E$ with a means of keeping track of approximate elapsed time during the response period. In addition to recording each response, $E$ noted the number of metronome beats that occurred between the last presented number of the series and the response, thus providing an approximate measure of response time.

\section{RESULTS}

A summary of the error rates for each experimental condition is given in Table 1. An overall analysis of variance performed on these data indicates the main effects of both experimental variables and their interaction to be significant: input, $F(1,56)=112.6$, $\mathrm{p}<.001$; response, $\mathrm{F}(1,56)=16.4$, $p<.001$; interaction, $F(1,56)=14.0$, $p<.001$. Mean response times for correct and incorrect responses combined are also presented in Table 1. Ss who were allowed a 5-sec interval after the series to respond did take significantly longer to respond than did those allowed 1 sec: slow input, $t(28)=8.4, p<.001 ;$ fast input, $t(28)=8.5, p<.001$.

While the error data of Table 1 reveal clear effects of both experimental variables, an analysis of these errors suggests that the principal distinction occurs between the slow and the fast response groups. A presentation of the error data in terms of error proximity is given in Fig. 1 .
The abscissa represents the distance between an incorrect and the correct response. For instance, if the digit 1 were the correct response on a given trial, the digits 0 and 2 both represent a proximity value of 1 ; the digits 3 and 4 are assigned a value of 2 , and so forth (Buschke \& Hinrichs, 1968). A trend analysis was performed on these error proximity functions using orthogonal polynomials (Winer, 1962); the linear components of the two fast response conditions accounted for a significant portion of the variance $(p<.001)$, while those of the two slow response conditions did not $(p>.05)$. In addition, the average slopes of these linear components for the fast response conditions were greater by a factor of 4 than those for the slow response conditions.

\section{DISCUSSION}

One of the principal findings of this study has been that the error proximity functions for the two fast response conditions differ considerably from those of the slow response conditions. When $S$ is required to identify the missing number within only $1 \mathrm{sec}$ following presentation of the series, the error distributions as a function of relative ordinal position show linear components that account for a significant portion of the variance, and these linear components have steep negative slopes. In the case of a 5-sec response criterion, however, no such significant linear components are found. In addition, all four of these curves of Fig. 1 exhibit some degree of "bowing"-that is, in all curves, the greatest number of errors occurs in Relative Positions 2 or 3 . There are two considerations suggesting that the above findings do not fit credibly with the notion that S's strategy is based upon the ordinal properties of the stimuli.

First, for any plausible method of processing that makes use of ordinal information, clearly the greatest number of errors should occur closest to the correct answer, with a considerable decrease in errors occurring in Relative Ordinal Positions 2 and 3 . In the curves obtained, however, most of the error decline takes place in Positions 4 and 5 , and the greatest number of errors never occurs in Position 1.

Second, as Buschke and Lenon (1969) indicate, an ordinal strategy would most likely occur when $S$ had ample time to reorganize stimuli as they were presented-in other words, when the input rate was slow. They report finding negative slopes in an earlier study at $1 / \mathrm{sec}$ and $2 / \mathrm{sec}$ presentation rates, but not at a $4 / \mathrm{sec}$ rate. The present data, however, reveal 
no systematic relationship between input rates and the slopes of the proximity distributions. Instead, it appears that output rate is the variable affecting these slopes, with a fast output resulting in a greater error gradient. This finding is difficult to explain in terms of an ordinal strategy.

Since the obtained results do not fit well with the concept of an ordinal strategy, an alternative explanation favored by the present authors is that the error proximity functions reflect the operation of a guessing bias in favor of the numbers in the middle of the ordinal sequence and against numbers at the ends. A similar bias has been reported by Restle (1964) in a paired-associates experiment involving numerical responses to nonsense syllables. Such a bias can, in fact, produce functions similar to those of the fast output conditions in Fig. 1. For example, a bias against reporting digits at the extremes of the series will be reflected in a low percentage of errors for Relative Ordinal Position 5, since such an error always involves either the digit 0 or the digit 9 . Similarly, an error at Position 3 usually involves the digits $3,4,5$, or 6 , and only infrequently digits at the ends of the series. Further, it is reasonable to expect that if errors are distributed according to a response bias, this effect should be more pronounced in the fast response conditions. In these conditions, if $\mathbf{S}$ cannot supply the missing digit immediately after presentation of a series, he will be forced to hazard a guess quickly, and such time-constrained guesses are unlikely to be distributed randomly (Warren \& Morin, 1965; Tune, 1964).

Evidence supporting a response bias hypothesis is presented in Fig. 2. This figure displays error proximity functions averaged over input conditions, and the corresponding functions predicted by a response bias hypothesis. These predicted curves were calculated on the basis of the total number of times each digit was incorrectly given, assuming that the probability of responding with that digit was independent of the correct answer on a particular trial. The similarity of these response bias distributions to the curves actually obtained strongly suggests that $S$ 's errors are characterized by an overall bias to respond with some numbers more often than with others, rather than by a tendency to respond with a number that is numerically close to the correct answer.

Two differences between the present experiment and those of Buschke and his colleagues may possibly account for our failure to find clearcut evidence of an ordinal

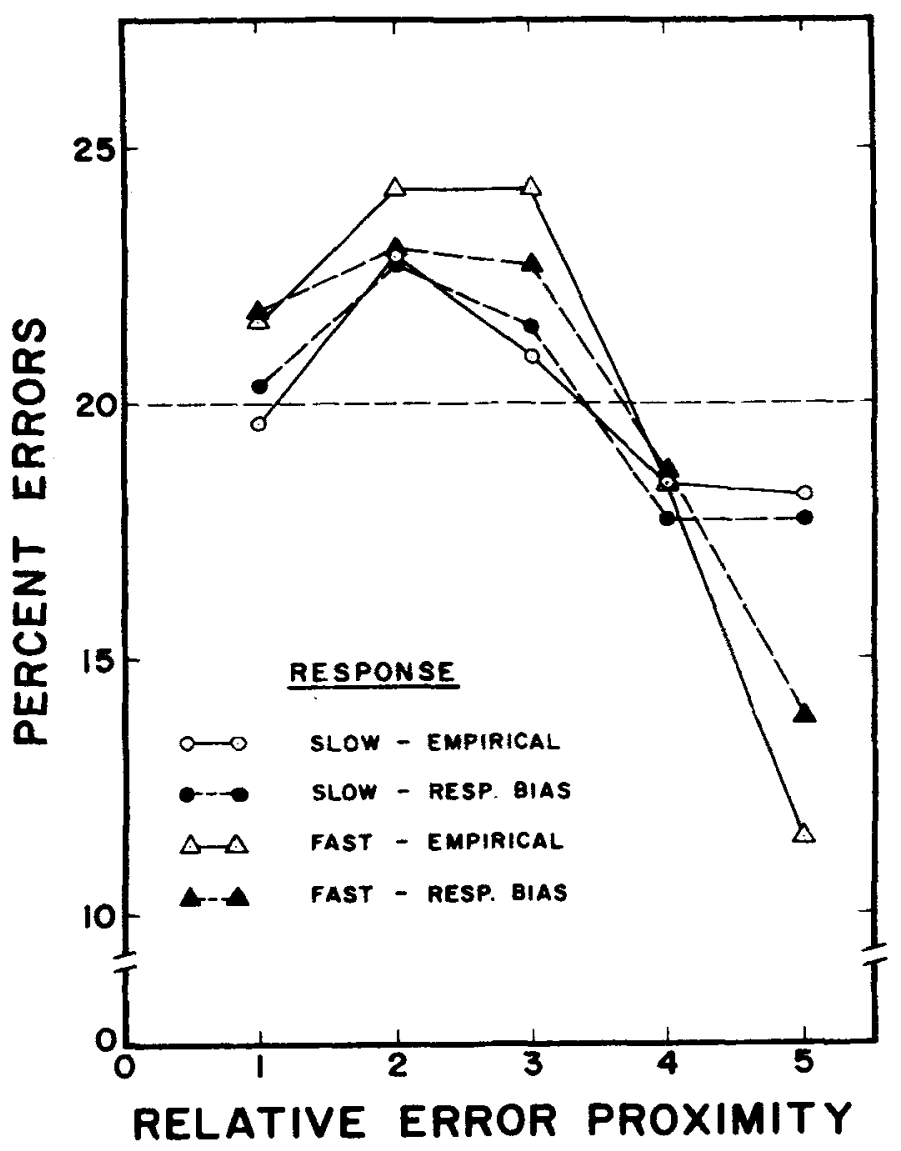

Fig. 2. Empirical error proximity functions and calculated response bias functions for the slow and fast response conditions.

strategy. First, the present study made use of a 10-item set instead of the 13 -item set usually employed. In this regard, it has been established (e.g., Buschke \& Lim, 1967) that items presented more recently tend to be given less often as errors. Such a recency effect would account for a greater proportion of items in a 10 than in a 13-item list, and might tend to obscure the operation of an ordinal strategy. A second difference relates to the homogeneity and familiarity of the set of digits $0-9$. Since this set is highly familiar and consists of one-digit numbers exclusively, $S$ may find it unnecessary to resort to a reorganization of the stimuli on the basis of their ordinal characteristics.

Finally, it is interesting to speculate on the type of strategy that may be employed to identify successfully the item that is absent, if $\mathbf{S}$ does not in fact utilize the ordinal properties of numbers. One plausible strategy, consistent with our data and supported by Ss' subjective reports, involves the use of an "anticipation" method. With this procedure, after approximately half the items have been presented, the $S$ selects perhaps two of the remaining alternatives and listens specifically for them. If either of these is not presented as part of the series, $S$ is able to respond correctly. If, however, both are presented, then $S$ must make the best possible guess in the amount of time available to him. With the slow input rate, accuracy of performance is unaffected by response time constraints. The extra second of response time utilized in the slow response group may only result in a more random distribution of guesses.

With an extremely rapid input rate, an anticipation strategy would be difficult to implement. Under such conditions, $\mathbf{S}$ may simply place all items in short-term storage, then, following presentation of the list, test successive hypotheses against the items in memory. In such a situation, response time limitations would be of considerable importance and are reflected in the $21 \%$ difference between the fast and slow response conditions.

\section{REFERENCES}

AARONSON, D. Temporal factors in perception and short-term memory. Psychological Bulletin, 1967, 67, $130-144$.

AVERBACH, E., \& CORIELL, A. S. Short term memory in vision. The Bell System 
Technical Journal, 1961, 40, 309-328.

BUSCHKE, $H$. Retention in immediate memory estimated without retrieval. Science, 1963a, 140, 56-57.

BUSCHKE, H. Relative retention in immediate memory determined by the missing scan method. Nature, 1963b, 200, 1129-1130

BUSCHKE, H., \& HINRICHS, J. V. Relative vulnerability of item-information in short-term storage for the missing scan. Journal of Verbal Learning \& Verbal Behaviox, 1968, 7, 1043-1048.

BUSCH KE, H. \& LENON, R. Ordinal sequence in short-term retention of numbers. Journal of Experimental Psychology, 1969, 81, 201-203.

BUSCHKE, H., \& LIM, H. Temporal and interactional effects in short-term storage. Pexception \& Psychophysics, 1967, 2, 107-114.

MADSEN, M. C. \& DRUCKER, J. M. Immediate memory by missing scan and modified digit span. Psychonomic Science, 1966, 6, 283-284.

RESTLE, F. Sources of difficulty in learning paired associates. In $R$. C. Atkinson (Ed.), Studies in mathematical psychology. Stanford: Stanford psy chology. Stanford: Stan
University Press, 1964. Pp. 116-172.

SPERLING, G. The information available in brief visual presentations. Psychological Monographs, 1960, 74(11, Whole No. 498).

TUNE, G. S. A brief survey of variables that influence random-generation. Perceptual
\& Motor Skills, 1964, 18, 705-710.

WARREN, P. S., \& MORIN, R. E. Random generation: Number of symbols to be randomized and time per response. Psychonomic Science, 1965, 3, 557-558. WINER, B. J. Statistical principles in experimental design. New York: McGraw-Hill, 1962. Pp. 132-135.

\section{NOTE}

1. No signal was provided to indicate the end of presentation of a series; preliminary observations revealed that such a signal improved neither speed nor accuracy.

(Accepted for publication May 25, 1972.) 